\title{
Antihyperalgesia effect of AMP-activated protein kinase (AMPK) activators in a mouse model of postoperative pain
}

\author{
Vaskar Das, Jeffrey S Kroin, Mario Moric, Robert J McCarthy, ${ }^{\circledR}$ Asokumar Buvanendran
}

Department of Anesthesiology, Rush University Medical Center, Chicago, Illinois, USA

\section{Correspondence to} Dr Asokumar Buvanendran, Department of Anesthesiology, Rush University Medical Center, Chicago, IL 60612, USA Asokumar@aol.com

Received 26 April 2019 Revised 21 May 2019 Accepted 29 May 2019 Published Online First 21 June 2019

\section{Check for updates}

(C) American Society of Regional Anesthesia \& Pain Medicine 2019. No commercial re-use. See rights and permissions. Published by BMJ.

\section{To cite: Das $V$}

Kroin JS, Moric M, et al.

Reg Anesth Pain Med

2019:44:781-786.

\section{ABSTRACT}

Background and objectives AMP-activated protein kinase (AMPK) activator drugs decrease hypersensitivity in mice with pain. This study examines if postsurgery treatment with the prototype AMPK activator metformin and a new mechanism-specific AMPK activator, 0304, after plantar hindpaw incision in mice, would reduce mechanical hypersensitivity and produce changes in the AMPK pathway in the dorsal root ganglion (DRG).

Methods To create postoperative pain, an incision was made in the left plantar hindpaw. Animals were randomized into four oral gavage drug treatment groups ( $n=8 /$ group): (1) vehicle, (2) metformin $200 \mathrm{mg} /$ kg, (3) $0304200 \mathrm{mg} / \mathrm{kg}$ and (4) $0304200 \mathrm{mg} / \mathrm{kg}$ plus metformin $200 \mathrm{mg} / \mathrm{kg}$. Drug gavages were performed 4 hours postsurgery and were repeated for 3 days. Mechanical hypersensitivity was measured with von Frey filaments. Changes in phosphorylated AMPactivated protein kinase alpha subunit, phosphorylated mechanistic target of rapamycin and phosphorylated eukaryotic initiation factor 2 alpha in DRG neurons were examined by immunohistochemistry.

Results 0304 or metformin increased von Frey thresholds (reduced mechanical hypersensitivity) in plantar incision mice versus vehicle-treated incision mice between days 1 and 4 (difference of mean area under the curve, $0304: 2.24 \mathrm{~g}^{*}$ day; $95 \% \mathrm{Cl}$ of the difference 0.28 to $4.21, p=0.011$; metformin: $2.56 \mathrm{~g}^{*}$ day; $95 \% \mathrm{Cl}$ of the difference 1.71 to $3.41, p<0.001)$. The drug combination further elevated von Frey thresholds. In the vehicle-treated group, the AMP-activated protein kinase alpha subunit was downregulated and mechanistic target of rapamycin and eukaryotic initiation factor 2 alpha were upregulated in DRG neurons; these deficits were reversed by the AMPK activator treatments.

Conclusions Early treatment with the mechanismspecific AMPK activator 0304 or the prototype AMPK activator metformin reduces mechanical hypersensitivity in a postoperative pain model in mice. These drugs also normalize the AMPK pathway in the DRG.

\section{INTRODUCTION}

With the current opioid crisis in the USA, the need to have non-opioid pharmacological therapy is critical. Therefore, an early treatment with a relatively safe non-opioid drug that is effective in treating postoperative pain would be an important innovation.

AMP-activated protein kinase (AMPK) is a key cellular regulatory system that ensures that ATP production and consumption are maintained in balance. ${ }^{1}$ When pathological conditions cause ATP levels to fall, AMPK is activated to restore cellular energy homeostasis. ${ }^{2}$ A previous plantar incision pain study in mice demonstrated that the prototype AMPK activator drug metformin $200 \mathrm{mg} / \mathrm{kg}$ administered preemptively starting 2 days presurgery and continued through 1 day postsurgery reduced postoperative mechanical hypersensitivity. ${ }^{3}$ Although the primary mode of AMPK activation with metformin is inhibition of complex 1 of the mitochondrial respiratory chain and suppression of ATP production, ${ }^{124}$ metformin can activate AMPK through multiple indirect mechanisms. ${ }^{3-6}$ In addition, metformin has other activities not involving AMPK,${ }^{47}$ and that may contribute to its antihyperalgesic activity in mice. Metformin is derived from a natural product used in herbal medicine and was not designed to target a specific pathway. ${ }^{4}$ Because metformin may have multiple AMPK-dependent mechanisms and AMPK-independent mechanisms of action, it is problematic to translate the animal studies to a clinical treatment. As an example, metformin reduced mechanical allodynia in a sciatic nerve injury model of neuropathic pain ${ }^{8}$ but did not reduce global pain scores in diabetic patients with neuropathic pain. ${ }^{9}$

$\mathrm{O} 304$ is a new direct AMPK activator that has concluded a phase IIa clinical trial in Europe for glucose homeostasis in patients with type 2 diabetes (metformin being the gold standard drug for treatment of type two diabetes) and for microvascular perfusion in skeletal muscle. ${ }^{10} \mathrm{O} 304$ suppresses the dephosphorylation of the Thr172 in the $\alpha$-subunit of AMPK by protein phosphatase 2C (PP2C) without inhibiting PP2C activity, a key regulatory mechanism to increase AMPK activity. ${ }^{2}{ }^{10}$ With a known mechanism of action, it is more likely that a successful O304 treatment in rodents will correspond to a therapeutic effect in humans. One pharmacokinetic difference between metformin and O304 is that metformin can cross the blood-brain barrier, ${ }^{11} 12$ while $\mathrm{O} 304$ is orally available but does not cross the blood-brain barrier. ${ }^{10}$ Therefore, the dorsal root ganglion (DRG) would be a common site to compare the activity of the two drugs on the AMPK pathway.

The purpose of our study was to demonstrate that early short-term treatment with $\mathrm{O} 304$ or metformin will reduce pain in a mouse plantar hindpaw incision model of postoperative pain. Since neither O304 nor metformin produces hypoglycemia in normal subjects or rodents, ${ }^{13-15}$ this can 
be considered a low-risk treatment. In, addition, we will also examine how the AMPK and stress response eukaryotic initiation factor 2 alpha pathways in the DRG are affected by the surgery and the postoperative pain treatment by these drugs.

\section{METHODS}

Postoperative pain model

All animal surgical procedures and protocols were approved by the Institutional Animal Use and Care Committee of Rush University Medical Center and adhered to the Animal Research: Reporting of In Vivo Experiments guidelines and the Guide for Care and Use of Laboratory Animals (1996). Experiments were performed on 32 young adult $20 \mathrm{~g}$ female CD-1 mice (Charles River, Wilmington, Maine, USA), housed four per cage before and after surgery. The choice of female mice was based only on the ease of housing (avoids injurious fighting between multihoused male mice). To create postoperative pain, mice were anesthetized with $1.5 \%$ isoflurane in oxygen. Under aseptic conditions, a $5 \mathrm{~mm}$ long skin incision was made in the left plantar hindpaw with a fresh \#11 blade. The incision was started (at the toe pads), $7 \mathrm{~mm}$ from the proximal edge of the heel, and extended $5 \mathrm{~mm}$ toward the heel. The skin was then gently retracted with a fine-tip toothpick to expose the underlying muscle. The underlying muscle was transiently elevated with fine forceps, leaving the muscle origin and insertion intact. The skin was closed with a single 7-0 mattress suture. This incision has been shown to produce mechanical hyperalgesia lasting 3-4 days in mice. ${ }^{16} 17$

\section{Postoperative drug treatment}

At 4 hours after surgery, the animals were evaluated for left (ipsilateral) von Frey mechanical hyperalgesia and were allocated to four oral gavage treatment groups (having similar low von Frey thresholds, about $0.5 \mathrm{~g}$ ): group 1: methyl cellulose (MC) vehicle $(n=8)$; group 2: metformin $200 \mathrm{mg} / \mathrm{kg}$ alone $(n=8)$; group 3: O304 $200 \mathrm{mg} / \mathrm{kg}$ alone $(\mathrm{n}=8)$ and group 4: combined drugs, O304 $200 \mathrm{mg} / \mathrm{kg}$ plus metformin $200 \mathrm{mg} / \mathrm{kg}(\mathrm{n}=8)$. Then, the first drug gavage was performed, with a 20-gage animal feeding needle (Popper, New Hyde Park, NY, USA) under light isoflurane anesthesia (to minimize trauma to the esophagus or airway). Drug gavages were for three more consecutive days, with von Frey testing 24 hours after each oral administration. If O304 or metformin has efficacy in a postoperative pain model, then the O304 group and the metformin group, or the drug combination, will have higher von Frey threshold values than the vehicle-treated group on postoperative days $1-4$.

The $200 \mathrm{mg} / \mathrm{kg}$ daily metformin dose was based on Burton et al's study on treating plantar incision postoperative pain in mice. Doses of $150 \mathrm{mg} / \mathrm{kg}$ or less were ineffective. Using US Food and Drug Administration (FDA) allometric scaling, the $200 \mathrm{mg} / \mathrm{kg}$ mouse dose is equivalent to $1000 \mathrm{mg} /$ day in humans, which is a typical starting dose for the use of metformin in type 2 diabetes. ${ }^{18}$ In Steneberg et al, ${ }^{10}$ the dose of 0304 used in the phase IIa clinical trial was $1000 \mathrm{mg}$ /day. Using US FDA allometric scaling, that is equivalent to $200 \mathrm{mg} / \mathrm{kg}$ daily $\mathrm{O} 304$ dose in mice. So, the $200 \mathrm{mg} / \mathrm{kg} \mathrm{O} 304$ dose was used in mice for O304 alone and when combined with metformin (since in the phase IIa trial, patients were also receiving metformin along with O304). ${ }^{10}$

\section{Postoperative pain-related behavioral measure}

Prior to surgery, animals were evaluated for mechanical hyperalgesia based on foot withdrawal from a mechanical stimulus. The tactile stimulus was a graded set (in grams) of von Frey nylon filaments $(0.028-5.50 \mathrm{~g})$. While the animal is standing on a grid, the filament was directed onto the plantar surface of the left (ipsilateral) hindpaw. The gram force producing withdrawal of the hindpaw is an indication of the nociceptive response of the animal. The gram force threshold was assessed using an iterative up-down method. ${ }^{19}$ In this paradigm, a lower force-withdrawal threshold means increased mechanical hypersensitivity. At presurgery, mice have a force-withdrawal threshold of about $3.5 \mathrm{~g}$.

\section{Drugs for oral gavage}

The drug vehicle of 2\% MC (M7027; Sigma-Aldrich, St. Louis, Missouri, USA) was formulated by adding $1.2 \mathrm{~g} \mathrm{MC}$ powder to $60 \mathrm{~mL}$ cold phosphate-buffered saline (PBS) pH 7.4 (Fisher Scientific, Pittsburgh, Pennsylvania, USA) and stirring vigorously on a magnetic stirrer until clear. Metformin was formulated by adding $240 \mathrm{mg}$ of metformin hydrochloride (Cayman Chemical, Ann Arbor, Michigan, USA) to $60 \mathrm{~mL}$ cold MC solution and stirring vigorously on a magnetic stirrer until clear, to a final concentration of $4 \mathrm{mg} / \mathrm{mL}$. O304 was formulated by adding $240 \mathrm{mg}$ of O304 (gift from Baltic Bio AB, Umeå, Sweden) to $60 \mathrm{~mL}$ cold $\mathrm{MC}$ solution and stirring vigorously on a magnetic stirrer for 60 min to produce a white suspension at a final concentration of 4 $\mathrm{mg} / \mathrm{mL}$. The combined drugs were formulated by adding $240 \mathrm{mg}$ of metformin hydrochloride and $240 \mathrm{mg}$ of $\mathrm{O} 304$ to $60 \mathrm{~mL}$ cold MC solution and stirring vigorously on a magnetic stirrer for 60 min to produce a white suspension at a final concentration of 4 $\mathrm{mg} / \mathrm{mL}$ for each drug. A daily oral gavage of $1.0 \mathrm{~mL}$ delivers 4 $\mathrm{mg}$ of $\mathrm{O} 304 \mathrm{and} /$ or metformin, which is $200 \mathrm{mg} / \mathrm{kg}$ in a $0.02 \mathrm{~kg}$ mouse.

\section{Immunohistochemistry (IHC) of DRG}

IHC was performed on DRG tissues at 24 hours after the fourth drug gavage (4 days postsurgery) in 20 additional mice (16 plantar hindpaw incision mice with four oral gavage drug groups treated for 4 days and four non-incision non-gavage naive controls). The mice were euthanized with carbon dioxide and immediately perfused transcardially with $0.9 \%$ saline followed by $4 \%$ paraformaldehyde. Ipsilateral lumbar L4-L6 DRGs were removed, postfixed in $4 \%$ paraformaldehyde for 48 hours and prepared for IHC in paraffin-mixed solutions. ${ }^{20} 21$ DRG samples were cut at $5 \mu \mathrm{m}$ thickness, deparaffinized with xylene and ethanol, and washed with deionized water. Thereafter, the sections were microwaved in a retrieval solution (10 mmol/L citrate, $\mathrm{pH}$ 6.0; Fisher Scientific) and sections were permeabilized with $0.5 \%$ Triton X-100 solution (Sigma-Aldrich) for $5 \mathrm{~min}$; and non-specific binding was blocked by incubation in 5\% normal goat serum (Millipore Cat\#NS02L, Sigma-Aldrich) for $30 \mathrm{~min}$ at room temperature. After that, DRG sections were incubated overnight with anti-mouse phosphorylated AMP-activated protein kinase alpha subunit (p-AMPK $\alpha$ ) antibody (1:100, p-AMPK $\alpha 1$ (phospho S487), Cat\#131357; Abcam, Cambridge, Massachusetts, USA), anti-mouse phosphorylated mechanistic target of rapamycin (p-mTOR) antibody $(1: 100$, p-mTOR (phospho S2448), Cat\#109268; Abcam), anti-mouse phosphorylated eukaryotic initiation factor 2 alpha (p-eIF2 $\alpha$ ) antibody (1:200, p-EIF2S1 (phospho S51), Cat\#32157; Abcam) and anti-mouse neuronal nuclei (NeuN) antibody (1:500, Millipore MAB377; Sigma-Aldrich). The samples were then incubated with the appropriate secondary antibody (diluted in PBS) for an hour at room temperature in a humidified dark chamber, including Alexa Fluor 555 (1:250, rabbit anti-mouse, Invitrogen Cat\#A21427; Fisher Scientific) and Alexa Fluor 488 (1:250, goat anti-rabbit, Invitrogen Cat\#A11008; Fisher Scientific). 


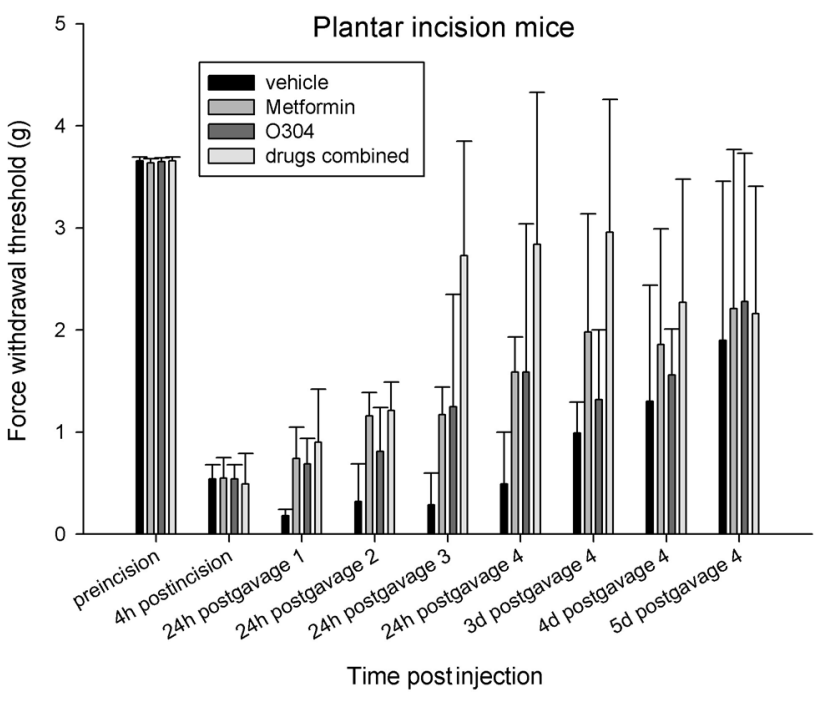

Figure 1 Ipsilateral von Frey force-withdrawal thresholds (g) over study days. Repeated measures over days 1-4 postsurgery demonstrated that metformin or 0304 had higher thresholds than vehicle and that the combination of the two drugs had the highest threshold. $n=8 /$ group. Data plotted as mean, SD.

Sections were washed with PBS and incubated with 4',6-diamidino-2-phenylindole (1:1000 dilution of $5 \mathrm{mg} / \mathrm{mL}$ stock, Sigma-Aldrich) for $5 \mathrm{~min}$. After subsequent washes with PBS, sections were cover-slipped with Fluoromount mounting medium (Novus, Centennial, Colorado, USA) at room temperature in the dark. Immunoreactivity was examined using a fluorescence microscope, and sections were visualized using a Nikon Eclepase microscope (Nikon, Melville, New York, USA). Images $(\times 200$, NIS-Elements software, V.D4.30.01; Nikon) were acquired using a Nikon Eclepase camera (DS-Ri2, Nikon) on the next day using the same camera settings. For each DRG section, the number of immunoreactive neurons was counted using Image J software V.1.8.0_60 (open source from National Institutes of Health, https://imagej.nih.gov/ij). Each animal had three to four sections from the DRG that were examined for IHC analysis. A similar technique was used to quantify the colocalization of biomarkers in neurons in the DRG of mice with tibia fracture. ${ }^{20} 21$

\section{Statistical analysis}

The distribution of the primary outcome was evaluated for normality using the Shapiro-Wilk test and for homogeneity of variance using the Levene test. The primary outcome of the study, the area under the curve (AUC) of the von Frey force thresholds over time (gram*day) between postoperative days 1-4, was compared between the four groups using the Kruskal-Wallis $\mathrm{H}$-test and post hoc non-parametric relative effects based on global rankings computed using a multivariate normal distribution, with CIs and $\mathrm{p}$ values adjusted for multiple comparisons using the Tukey method. von Frey force thresholds over time were compared between the four groups using a linear mixed effects model with repeated measures and an autoregressive lag 1 covariance structure. Post hoc comparisons were controlled for error inflation using the Holm-Bonferroni method. Day 0 baseline measures were compared among the groups with a linear mixed-method model test of fixed effects. For the IHC of DRGs, differences in percent neurons labeled in the DRG between the five groups of mice were compared with analysis of variance with post hoc Tukey test with adjustment for multiple comparisons. Data were analyzed using RStudio V.1.2.1335
(Integrated Development for R. RStudio, Boston, Massachusetts, USA; http://www.rstudio.com/) and R V.3.6, release date April 26, 2019 (The R Foundation for Statistical Computing, Vienna, Austria) and SAS V.9.4 software.

The sample size for demonstrating that metformin elevated von Frey thresholds in postoperative incision pain mice versus vehicle-treated incision mice over the postoperative period was obtained from Burton $e t a l^{3}$ in the plantar incision model, in which metformin $200 \mathrm{mg} / \mathrm{kg}$ administered starting 2 days presurgery and continued through 1 day postsurgery showed elevated von Frey thresholds compared with vehicle-treated mice. That mouse study had five groups, with $\mathrm{n}=6$ per group, and so we chose $n=8$ per group for our behavioral study.

\section{RESULTS}

\section{Plantar incision model pain following AMPK activator} treatment

No animals had any adverse events during the 9-day behavioral drug study. Prior to hindpaw incision (day 0), ipsilateral von Frey force-withdrawal thresholds $(p=0.71)$ were similar among the four experimental groups (figure 1). At 4 hours after hindpaw incision (day 0), ipsilateral von Frey force-withdrawal thresholds $(p=0.95)$ were similar among the four experimental groups.

The mean von Frey threshold per study day is shown in figure 1 . The mixed repeated measures model between days 1 and 4 showed that $\mathrm{O} 304$ or metformin gavage elevated von Frey thresholds compared with vehicle-treated mice, and that the von Frey thresholds of $\mathrm{O} 304$ plus metformin mice were greater than the O304-treated mice or the metformin-treated mice; all comparisons had a $\mathrm{p}$ value of $<0.05$.

The mean AUC of the von Frey force-withdrawal threshold by time between days 1 and 4 for hindpaw incision mice treated with vehicle was $0.95 \mathrm{~g}$ *day (95\% CI -0.24 to 2.14 ) (figure 2). O304 oral gavage elevated von Frey thresholds (reduced mechanical hyperalgesia) compared with the vehicle-treated mice with a difference of mean AUC of $2.24 \mathrm{~g}$ *day $(95 \% \mathrm{CI}$ 0.28 to $4.21, p=0.011)$. Metformin gavage elevated von Frey thresholds compared with vehicle-treated mice with a difference of mean AUC of $2.56 \mathrm{~g}$ *day (95\% CI 1.71 to 3.41, p<0.001). von Frey force-withdrawal thresholds were similar between the metformin and $\mathrm{O} 304$ groups $(p=0.94)$. The AUC of the O304 plus metformin mice trended toward being greater than the O304-treated mice with a difference of mean AUC of 2.62 g*day $(95 \%$ CI 0.27 to $4.96, \mathrm{p}=0.053)$ or trended toward being greater than the metformin-treated mice with a difference of mean AUC of $2.31 \mathrm{~g}$ *day (95\% CI 0.54 to $4.07, \mathrm{p}=0.068$ ); the AUC of $\mathrm{O} 304$ plus metformin mice was higher than the vehicle-treated mice, with a difference of mean AUC of $4.86 \mathrm{~g}$ *day (95\% CI 3.05 to $6.67, \mathrm{p}<0.001)$.

\section{IHC of DRG of plantar incision pain mice}

Immunostaining of pain-related biomarkers (colocalized in neurons, NeuN) in the DRG is shown for five groups of mice (a new experiment with four incision groups treated for 4 days, plus one naive control group, euthanized at 4 days postsurgery; total $\mathrm{n}=20$ ) in figure 3. Quantitative analysis of immunohistochemical staining is shown in figure 4. The difference in percent of neurons in the L4-L6 DRG expressing p-AMPK $\alpha$ activity in the incision vehicle-treated animals compared with the naive mice was decreased by $64.3 \%$ (95\% CI $44.6 \%$ to $84.0 \%, \mathrm{p}<0.001$ ). Treatment with $\mathrm{O} 304$, metformin or $\mathrm{O} 304$ plus metformin increased the percent of DRG neurons with p-AMPK $\alpha$ expression by $51.7 \%$ (95\% CI $32.2 \%$ to $71.3 \%, \mathrm{p}<0.001$ ), $60.4 \%$ 
Plantar incision mice

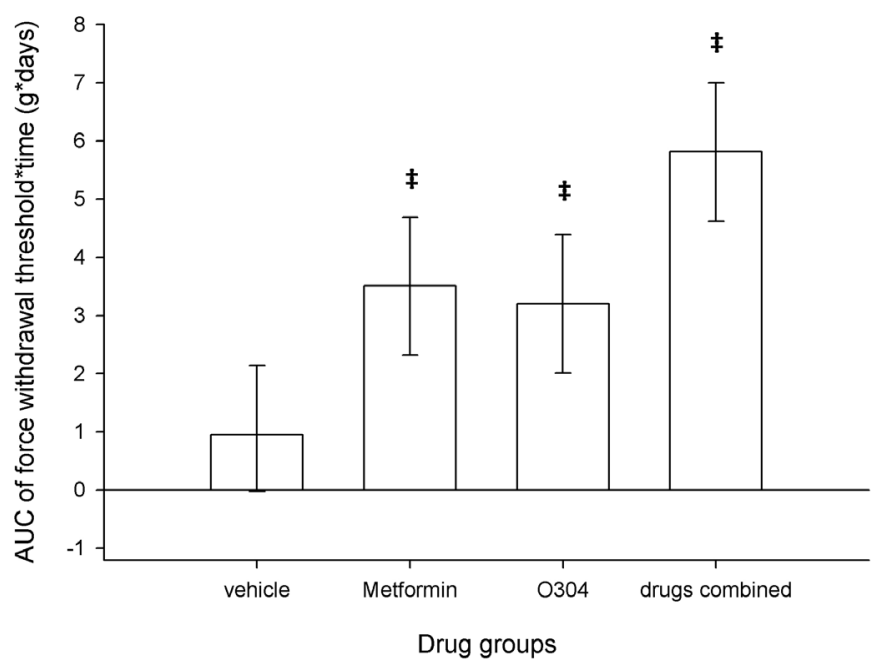

Figure 2 AUC of ipsilateral von Frey force-withdrawal thresholds (gram per day) over days 1-4 postsurgery. Vehicle-treated mice had the lowest AUC (greatest hyperalgesia), while metformin and/or 0304 elevated the AUC (pain relief). $¥ D$ Different from vehicle. Data plotted as mean, $95 \% \mathrm{Cl}$. $\mathrm{n}=8 /$ group. AUC, area under the curve.

(95\% CI $40.8 \%$ to $80.1 \%, \mathrm{p}<0.001)$ or $58.8 \%$ (95\% CI $39.1 \%$ to $78.4 \%, \mathrm{p}<0.001$ ), respectively, compared with vehicle-treated mice. The percent of neurons stained by $\mathrm{p}-\mathrm{AMPK} \alpha$ was similar among the three treatment groups and the naive group; all comparisons had a $\mathrm{p}$ value of $>0.32$. The difference in percent of neurons in the L4-L6 DRG expressing p-mTOR in the incision vehicle-treated animals compared with the naive mice was increased by $74.7 \%$ (95\% CI $59.7 \%$ to $89.7 \%$, p < 0.001 ). Treatment with $\mathrm{O} 304$, metformin or $\mathrm{O} 304$ plus metformin decreased the percent of DRG neurons with p-mTOR expression by $68.1 \%$ (95\% CI $53.1 \%$ to $83.0 \%, \mathrm{p}<0.001$ ), $59.5 \%$ (95\% CI $44.5 \%$ to $74.5 \%, \mathrm{p}<0.001$ ) or $66.8 \%$ (95\% CI $51.8 \%$ to $81.7 \%$, $\mathrm{p}<0.001$ ), respectively, compared with vehicle-treated mice. The percent of neurons stained by p-mTOR was similar among the O304 and O304 plus metformin treatment groups and the naive group; all comparisons had a $p$ value of $>0.42$. In the metformin-treated group, the percent was higher than that in the naive group $(\mathrm{p}=0.046)$.

The difference in percent of neurons in the L4-L6 DRG expressing p-eIF $2 \alpha$ in the incision vehicle-treated animals compared with the naive mice was increased $63.5 \%(95 \% \mathrm{CI}$ $40.5 \%$ to $86.5 \%, \mathrm{p}<0.001)$. Treatment with O304, metformin or O304 plus metformin decreased the percent of DRG neurons with p-eIF $2 \alpha$ expression by $53.2 \%$ (95\% CI $30.1 \%$ to $76.2 \%$, $\mathrm{p}<0.001$ ), $50.8 \%$ (95\% CI $27.8 \%$ to $73.8 \%, \mathrm{p}<0.001$ ) or $53.4 \%$ (95\% CI $30.3 \%$ to $76.4 \%, \mathrm{p}<0.001)$, respectively, compared with the vehicle-treated mice. The percent of neurons stained by p-eIF $2 \alpha$ was similar among the three treatment groups and the naive group; all comparisons had a p value of $>0.45$.

\section{DISCUSSION}

Postsurgical administration of $\mathrm{O} 304$ or metformin at $200 \mathrm{mg} /$ $\mathrm{kg}$ for 4 days reduced mechanical hyperalgesia in mice with plantar hindpaw incision. A previous metformin study in mice had shown similar results, although in that study, metformin was administered preemptively starting 2 days presurgery, ${ }^{3}$ while we began drug administration postsurgery when mechanical hyperalgesia had already developed. In our study, the combination
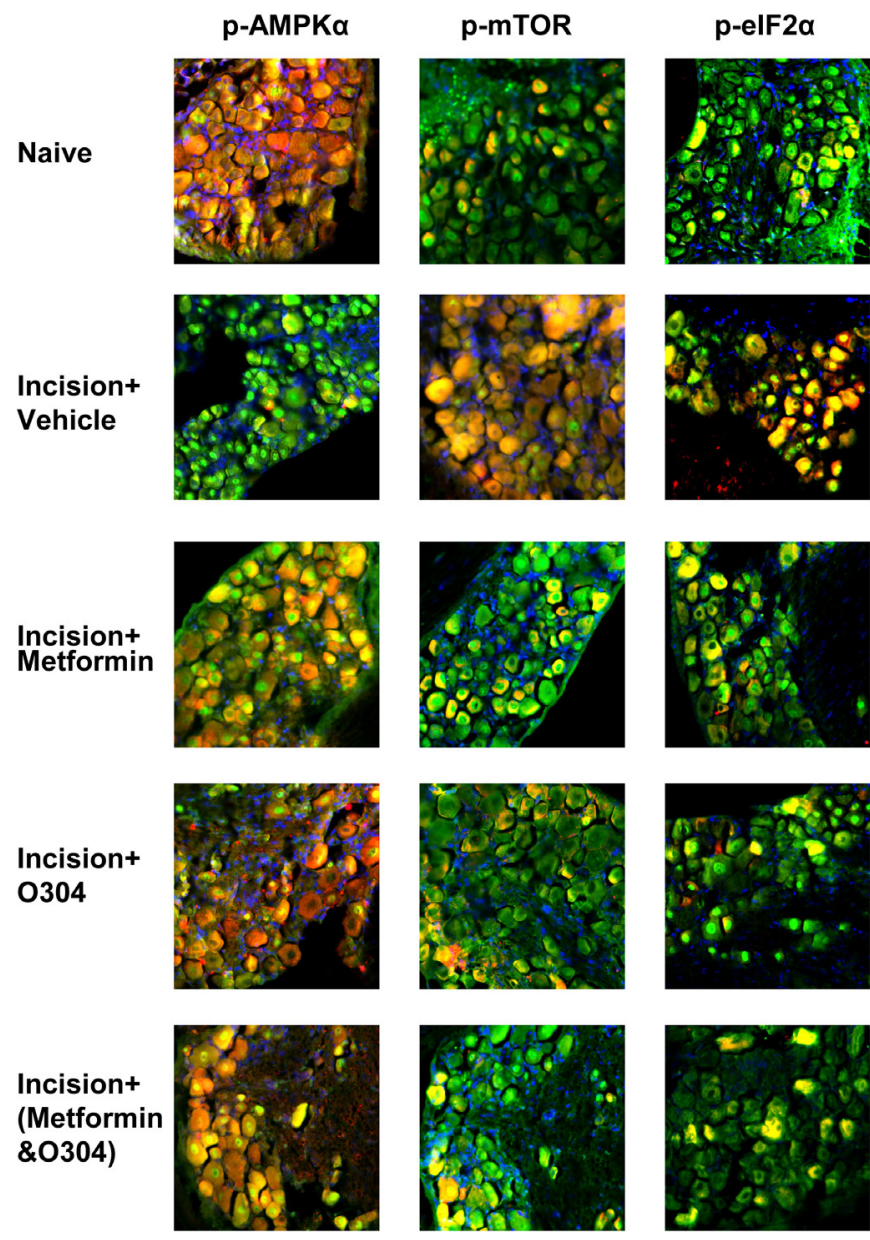

Figure 3 Immunohistochemistry of p-AMPK $\alpha, p$-mTOR and p-elF $2 \alpha$ (all biomarkers are red stains) in neurons (colocalization with neuronal nuclei (NeuN in green)) in ipsilateral lumbar dorsal root ganglia of mice with plantar hindpaw incision and euthanized at 4 days postsurgery. p-AMPK $\alpha$ staining is seen in virtually all neurons (appears yellow as red biomarker stain blends with green neuron stain) in naive mice, is greatly decreased in vehicle-treated incision mice (mostly green) and is restored (again yellow) in metformin, 0304 or 304 plus metformin incision mice. p-mTOR staining is seen in only a fraction of neurons (mostly green) in naive mice, is greatly increased in vehicle-treated mice (virtually all yellow) and again is seen in only a fraction of neurons (mostly green) in metformin, 0304 or 304 plus metformin mice. p-elF $2 \alpha$ staining is seen in only a fraction of neurons (mostly green) in naive mice, is greatly increased in vehicle-treated mice (virtually all yellow) and is restored (mostly green) in metformin, 0304 or 304 plus metformin mice. p-AMPK $\alpha$, phosphorylated AMP-activated protein kinase alpha subunit; p-mTOR, phosphorylated mechanistic target of rapamycin; p-elF $2 \alpha$, phosphorylated eukaryotic initiation factor 2 alpha.

of $\mathrm{O} 304200 \mathrm{mg} / \mathrm{kg}$ and metformin at $200 \mathrm{mg} / \mathrm{kg}$ was more potent than either drug alone in reducing hyperalgesia, and so future studies can explore if drug combinations are warranted. In a previous study from our group, with a mouse tibia fracture model of complex regional pain syndrome, metformin $200 \mathrm{mg} /$ $\mathrm{kg}$ administered after fracture reduced mechanical allodynia. ${ }^{21}$

Plantar hindpaw incision greatly reduced the percent of neurons in the DRGs that express p-AMPK $\alpha$. A similar reduction in AMP-activated protein kinase alpha subunit (AMPK $\alpha)$ was seen in the DRG of mice in a tibia fracture model of complex regional pain syndrome. ${ }^{21}$ In the present study, the AMPK activators metformin and $\mathrm{O} 304$, and their combination restored 
A

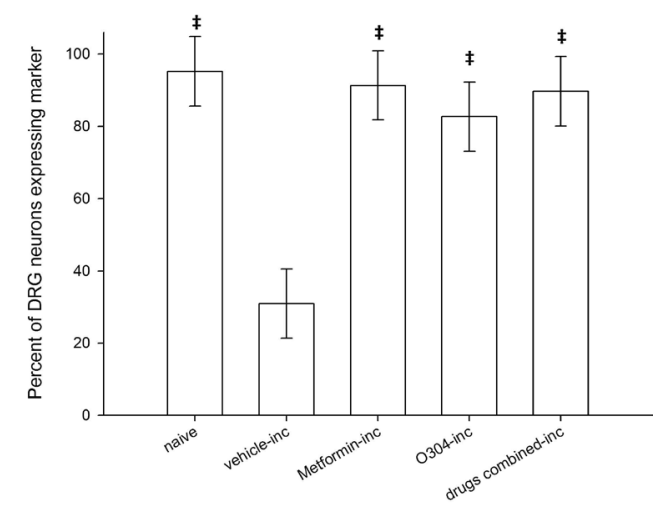

B

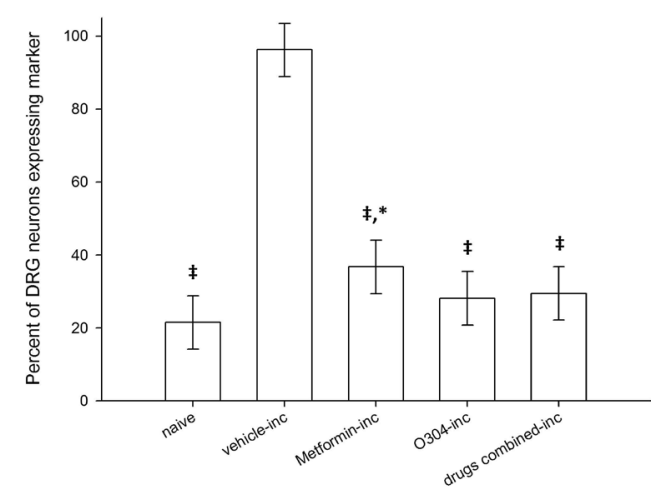

C

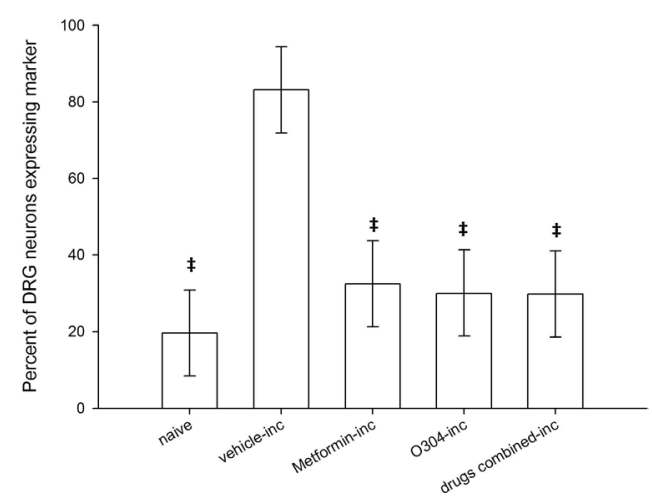

Figure 4 Change in neuron biomarkers with AMP-activated protein kinase activator treatment after plantar inc. Data are presented as percent of neurons expressing each biomarker in the ipsilateral lumbar dorsal root ganglia. (A) p-AMPK $\alpha$ was downregulated after plantar inc in the vehicle-treated group but was increased to normal levels (naive) by metformin, 0304 or 0304 plus metformin treatment for 4 days. $\ddagger$ Different from vehicle-treated inc mice, $p<0.001$. (B) $p$-mTOR was upregulated after plantar inc but was decreased to normal levels (naive) by 0304 or 0304 plus metformin treatment; and decreased by metformin treatment. $\ddagger$ Different from vehicle-treated inc mice, $p<0.001$. *Different from naive mice, $p<0.05$. (C) $p$-elF2 $\alpha$ was upregulated after plantar inc but was decreased to normal levels (naive) by metformin, 0304 or 0304 plus metformin. ¥Different from vehicle-treated inc mice, $\mathrm{p}<0.001$. Data are plotted as mean, $95 \% \mathrm{Cl}$. $n=4$ /group. DRG, dorsal root ganglion; inc, incision; $\mathrm{p}-\mathrm{AMPK} \alpha$, phosphorylated AMP-activated protein kinase alpha subunit; $\mathrm{p}-\mathrm{MTOR}$, phosphorylated mechanistic target of rapamycin; p-elF2 $\alpha$, phosphorylated eukaryotic initiation factor 2 alpha.
p-AMPK $\alpha$ levels to normal (similar to naive mice). It has been suggested that one-way AMPK re-establishes energy homeostasis is by turning off energy-consuming processes, such as the firing of action potentials in neurons, ${ }^{122}$ and so that may be the link between AMPK activation and reduction of hyperalgesia. As an example, AMPK directly phosphorylates the Kv2.1 potassium channel, which results in decreased firing of action potentials. ${ }^{23}$ At present, there are at least 100 target proteins of activated AMPK, ${ }^{2}$ and so AMPK may decrease mechanical hyperalgesia through multiple mechanisms.

In our study, plantar incision greatly increased the percent of neurons in the DRG that expressed p-mTOR. After nerve injury, p-mTOR was upregulated in rat DRG lysates. ${ }^{22}$ In the present study, AMPK activator treatment with O304, or the O304 plus metformin combination, restored p-mTOR to its low level (similar to naive mice), and metformin treatment lowered p-mTOR levels. Previous studies have demonstrated that AMPK activation inhibits the mechanistic target of rapamycin complex 1 (mTORC1) pathway. ${ }^{1222} 24$ Directly blocking the mTORC1 pathway with its inhibitor rapamycin in neuropathic mice can reduce mechanical hyperalgesia, but in normal mice, rapamycin increased mechanical hyperalgesia, ${ }^{8}$ so AMPK activator drugs are a better option.

Eukaryotic initiation factor 2 (eIF2) is an important integrator of cellular stress responses. ${ }^{25} 26$ In our study, plantar incision greatly increased the percent of neurons in the DRG that expressed p-eIF $2 \alpha$. In a mouse inflammatory pain model with mechanical hyperalgesia, p-eIF2 $\alpha$ was upregulated in DRG lysates, ${ }^{25}$ and in a rat diabetic neuropathy model with mechanical hyperalgesia, p-eIF $2 \alpha$ was upregulated in sciatic nerve axons. ${ }^{27}$ In the present study, AMPK activators, metformin and O304, and their combination restored p-eIF2 $\alpha$ levels to normal (similar to naive mice). However, the pathway linking p-eIF2 $\alpha$ and AMPK activation in peripheral neurons is unknown. In the mouse inflammatory pain model, decreasing elF $2 \alpha$ phosphorylation did not decrease mechanical hyperalgesia, ${ }^{25}$ but in the rat diabetic neuropathy study with cellular stress and upregulated p-elF $2 \alpha$, decreasing p-elF $2 \alpha$ did block mechanical hyperalgesia, ${ }^{27}$ so it is not clear if the downregulation of $\mathrm{p}-\mathrm{eIF} 2 \alpha$ by AMPK activators contributed to pain reduction in the mouse plantar incision model.

The doses of O304 and metformin used in this study (200 $\mathrm{mg} / \mathrm{kg}$ ) were chosen based on estimated safe starting doses in humans. Since both drugs are already in human use, they are attractive alternatives to opioids for managing postoperative pain. However, despite metformin being a widely used drug for type 2 diabetes for 40 years, it is questionable whether it reduces pain in humans. ${ }^{9}$ A 2013 retrospective chart review of patients with lumbar radiculopathy pain, comparing diabetic patients on metformin with a control group, found reduced pain scores with the metformin group. ${ }^{28}$ However, a 2015 prospective phone-based survey of metformin users (average dose of $1432 \mathrm{mg} /$ day) and non-users among type 2 diabetic patients found no difference in Brief Pain Inventory global pain scores. ${ }^{9}$ In addition, among those patients with neuropathic pain, there was no difference in global pain scores between the metformin and the non-metformin users. Nevertheless, no study has examined if metformin can reduce pain after surgery, and so a clinical trial may be warranted. In the past, metformin was withheld before surgery for fear of metformin-associated lactic acidosis. However, there is no evidence from prospective trials that metformin is associated with an increased risk of lactic acidosis, ${ }^{29}$ and guidelines have moved toward the continuation of metformin. ${ }^{30}$ In the last few years, site-specific AMPK 
activator compounds for type 2 diabetes have been synthesized. ${ }^{2}$ While issues of cardiac hypertrophy in preclinical studies have prevented some of these drugs from being released, one new drug, O304, does not cause increased heart weight in preclinical studies and has completed a phase IIa clinical trial in Sweden. ${ }^{210}$ O304 increases AMPK activity by suppressing the dephosphorylation of p-T172 AMPK $\alpha$ without inhibiting phosphatase PP2C, thus prolonging the active (phosphorylated) state. On the other hand, metformin may have multiple indirect mechanisms by which AMPK may be activated ${ }^{3-6}$ and many non-AMPK activities that may influence pain. ${ }^{47}$ Therefore, with a more specific mechanism of action, O304 may be a better candidate than metformin for a clinical trial in postoperative pain. Moreover, the average plasma half-life of metformin in men after oral treatment is 6 hours, ${ }^{18}$ whereas the plasma half-life of 0304 is about 15 -fold longer, ${ }^{10}$ possibly making $\mathrm{O} 304$ a better candidate for treatment of chronic pain conditions.

One limitation of our study is that we only tested a single dose of metformin and O304. Higher doses may confer greater benefit than that seen in the doses selected in this study. Our intention was to assess the early intervention with AMPK activators to modify pain after surgery. We selected a moderate dose of metformin, $200 \mathrm{mg} /$ day, that corresponds to a clinical dose of approximately $500 \mathrm{mg}$ two times per day. This dose was chosen because it has a high safety profile in humans, which increases the generalizability of our findings. The same dose of O304 was chosen, again because it corresponds to a clinical dose of approximately $1000 \mathrm{mg} /$ day, which was used in a phase IIa clinical trial. However, additional studies of varying doses and dosing schedules are warranted. Another limitation of our study is that we did not evaluate the role of off target effects on the efficacy of metformin in pain models. In addition, in mice models of nerve injury neuropathic pain, although there are several reasons for favoring a peripheral mechanism of action for metformin, ${ }^{8}$ inhibition of spinal cord dorsal horn microglial activation has also been implicated as a factor in reducing mechanical allodynia. ${ }^{31}$

In conclusion, the AMPK activator drugs metformin and $\mathrm{O} 304$ reduce mechanical hyperalgesia following surgery in mice. O304 represents a new class of drugs that targets a key physiological regulatory mechanism to increase AMPK activity. Considering the low side-effect profile of these compounds in humans, clinical trials for the short-term use of these drugs to manage postoperative pain should be considered.

Contributors VD, JSK, MM and AB contributed to the experimental design. VD and JSK performed the experiments. VD collected the experimental data. VD performed the immunohistochemistry. MM and RJM contributed to the statistical analysis. VD, JSK, RJM and AB drafted the manuscript. VD, JSK, MM, RJM and AB reviewed and approved the final manuscript.

Funding University Anesthesiologists, S.C., Chicago, IL.

Competing interests None declared.

Patient consent for publication Not required.

Ethics approval The Institutional Animal Use and Care Committee of Rush University Medical Center, Chicago, IL, USA.

Provenance and peer review Not commissioned; externally peer reviewed.

Data availability statement Data are available upon reasonable request.

\section{REFERENCES}

1 Hardie DG. Keeping the home fires burning: AMP-activated protein kinase. J $R$ Soc Interface 2018;15.
2 Steinberg GR, Carling D. AMP-activated protein kinase: the current landscape for drug development. Nat Rev Drug Discov 2019;280.

3 Burton MD, Tillu DV, Mazhar K, et al. Pharmacological activation of AMPK inhibits incision-evoked mechanical hypersensitivity and the development of hyperalgesic priming in mice. Neuroscience 2017;359:119-29.

4 Rena G, Hardie DG, Pearson ER. The mechanisms of action of metformin. Diabetologia 2017;60:1577-85.

5 Ouyang J, Parakhia RA, Ochs RS. Metformin activates AMP kinase through inhibition of AMP deaminase. J Biol Chem 2011;286:1-11.

6 Mejia GL, Asiedu MN, Hitoshi Y, et al. The potent, indirect adenosine monophosphateactivated protein kinase activator R419 attenuates mitogen-activated protein kinase signaling, inhibits nociceptor excitability, and reduces pain hypersensitivity in mice. Pain Rep 2016:1:e562.

7 Pryor R, Cabreiro F. Repurposing metformin: an old drug with new tricks in its binding pockets. Biochem J 2015:471:307-22.

8 Melemedjian OK, Khoutorsky A, Sorge RE, et al. MTORC1 inhibition induces pain via IRS-1-dependent feedback activation of ERK. Pain 2013:154:1080-91.

9 Smith B, Ang D. Metformin: potential analgesic? Pain Med 2015;16:2256-60.

10 Steneberg P, Lindahl E, Dahl U, et al. PAN-AMPK activator 0304 improves glucose homeostasis and microvascular perfusion in mice and type 2 diabetes patients. $\mathrm{JCl}$ Insight 2018;3:e99114.

11 Łabuzek K, Suchy D, Gabryel B, et al. Quantification of metformin by the HPLC method in brain regions, cerebrospinal fluid and plasma of rats treated with lipopolysaccharide. Pharmacol Rep 2010;62:956-65.

12 Koenig AM, Mechanic-Hamilton D, Xie SX, et al. Effects of the insulin sensitizer metformin in Alzheimer disease: pilot data from a randomized placebo-controlled crossover study. Alzheimer Dis Assoc Disord 2017;31:107-13.

13 Hermann LS. Metformin: a review of its pharmacological properties and therapeutic use. Diabete Metab 1979;5:233-45.

14 Marshall SM. 60 years of metformin use: a glance at the past and a look to the future. Diabetologia 2017;60:1561-5.

15 Ge A, Wang S, Miao B, et al. Effects of metformin on the expression of AMPK and STAT3 in the spinal dorsal horn of rats with neuropathic pain. Mol Med Rep 2018;17:5229-37.

16 Pogatzki EM, Raja SN. A mouse model of incisional pain. Anesthesiology 2003;99:1023-7.

17 Kroin JS, Das V, Moric M, et al. Efficacy of the ketamine metabolite (2R,6R)hydroxynorketamine in mice models of pain. Reg Anesth Pain Med 2019;44:111-7.

18 Bristol-Myers Squibb. Glucophage (metformin hydrochloride tablets) label information 2017. Available: https://packageinserts.bms.com/pi/pi_glucophage_xr.pdf [Accessed April 17, 2019].

19 Chaplan SR, Bach FW, Pogrel JW, et al. Quantitative assessment of tactile allodynia in the rat paw. J Neurosci Methods 1994;53:55-63.

20 Das V, Kroin JS, Moric M, et al. Biochemical and pharmacological characterization of a mice model of complex regional pain syndrome. Reg Anesth Pain Med 2017:42:507-16

21 Das V, Kroin JS, Moric M, et al. Early treatment with metformin in a mice model of complex regional pain syndrome reduces pain and edema. Anesth Analg 2019. doi:10.1213/ANE.0000000000004057. [Epub ahead of print: 18 Feb 2019].

22 Melemediian OK, Asiedu MN, Tillu DV, et al. Targeting adenosine monophosphateactivated protein kinase (AMPK) in preclinical models reveals a potential mechanism for the treatment of neuropathic pain. Mol Pain 2011;7.

23 Ikematsu N, Dallas ML, Ross FA, et al. Phosphorylation of the voltage-gated potassium channel Kv2.1 by AMP-activated protein kinase regulates membrane excitability. Proc Natl Acad Sci 2011;108:18132-7.

24 Tillu DV, Melemedjian OK, Asiedu MN, et al. Resveratrol engages AMPK to attenuate ERK and mTOR signaling in sensory neurons and inhibits incision-induced acute and chronic pain. Mol Pain 2012;8.

25 Khoutorsky A, Sorge RE, Prager-Khoutorsky M, et al. EIF2 $\alpha$ phosphorylation controls thermal nociception. Proc Natl Acad Sci U S A 2016;113:11949-54.

26 Megat S, Price TJ. Therapeutic opportunities for pain medicines via targeting of specific translation signaling mechanisms. Neurobiol Pain 2018;4:8-19.

27 Inceoglu B, Bettaieb A, Trindade da Silva CA, et al. Endoplasmic reticulum stress in the peripheral nervous system is a significant driver of neuropathic pain. Proc Natl Acad Sci U S A 2015;112:9082-7.

28 Taylor A, Westveld AH, Szkudlinska M, et al. The use of metformin is associated with decreased lumbar radiculopathy pain. J Pain Res 2013;6:755-63.

29 Salpeter SR, Greyber E, Pasternak GA, et al. Risk of fatal and nonfatal lactic acidosis with metformin use in type 2 diabetes mellitus. Cochrane Database Syst Rev 2010;4.

30 Hulst $\mathrm{AH}$, Polderman JAW, Ouweneel $\mathrm{E}$, et al. Peri-operative continuation of metformin does not improve glycaemic control in patients with type 2 diabetes: a randomized controlled trial. Diabetes Obes Metab 2018:20:749-52

31 Inyang KE, Szabo-Pardi T, Wentworth E, et al. The antidiabetic drug metformin prevents and reverses neuropathic pain and spinal cord microglial activation in male but not female mice. Pharmacol Res 2019:139:1-16. 